\title{
Effective Emission Tomography Image Reconstruction Algorithms for SPECT Data
}

\author{
J. Ramírez ${ }^{1}$, J.M. Górriz ${ }^{1}$, M. Gómez-Río ${ }^{2}$, A. Romero ${ }^{1}$, R. Chaves $^{1}$, \\ A. Lassl $^{1}$, A. Rodríguez ${ }^{2}$, C.G. Puntonet ${ }^{4}$, F. Theis ${ }^{5}$, and E. Lang ${ }^{3}$ \\ ${ }^{1}$ Dept. of Signal Theory, Networking and Communications, \\ University of Granada, Spain \\ javierrp@ugr.es \\ ${ }^{2}$ Servicio de Medicina Nuclear, \\ Hospital Universitario Virgen de las Nieves (HUVN), Granada, Spain \\ ${ }^{3}$ Institut für Biophysik und physikalische Biochemie, \\ University of Regensburg, Germany \\ ${ }^{4}$ Dept. of Architecture and Computer Technology, \\ University of Granada, Spain \\ ${ }^{5}$ Max Planck Institute for Dynamics and Self-Organisation, \\ Bernstein Center for Computational Neuroscience, Göttingen, Germany
}

\begin{abstract}
Medical image reconstruction from projections is computationally intensive task that demands solutions for reducing the processing delay in clinical diagnosis applications. This paper analyzes reconstruction methods combined with pre- and post-filtering for Single Photon Emission Computed Tomography (SPECT) in terms of convergence speed and image quality. The evaluation is performed by means of an image database taken from a concurrent study investigating the use of SPECT as a diagnostic tool for the early onset of Alzheimer-type dementia. Filtered backprojection (FBP) methods combined with frequency sampling 2D pre- and post-filtering provides a good trade-off between image quality and delay. Maximum likelihood expectation maximization (ML-EM) improves the quality of the reconstructed image but with a considerable increase in processing delay. To overcome this problem the ordered subsets expectation maximization (OS-EM) method is found to be an effective algorithm for reducing the computational cost with an image quality similar to ML-EM.
\end{abstract}

\section{Introduction}

Emission-computed tomography (ECT) has been widely employed in biomedical research and clinical medicine during the last three decades. ECT differs fundamentally from many other medical imaging modalities in that it produces a mapping of physiological functions as opposed to imaging anatomical structure.

Tomographic radiopharmaceutical imaging, or ECT, provides in vivo threedimensional maps of a pharmaceutical labeled with a gamma ray emitting radionuclide. The distribution of radionuclide concentrations are estimated from a set of projectional images acquired at many different angles around the patient. 
Single Photon Emission Computed Tomography (SPECT) imaging techniques employ radioisotopes which decay emitting predominantly a single gamma photon. This represents the fundamental difference between PET (Positron Emission Tomography) and SPECT. PET systems employ isotopes in which a couple of photons are produced in each individual annihilation. There is a rich variety of isotopes that decay, emitting a single-photon and which consequently can be utilized in SPECT. When the nucleus of a radioisotope disintegrates, a gamma photon is emitted with a random direction which is uniformly distributed in the sphere surrounding the nucleus. If the photon is unimpeded by a collision with electrons or other particles within the body, its trajectory will be a straight line or "ray". In order for a photon detector external to the patient to discriminate the direction that a ray is incident from, a physical collimation is required. Typically, lead collimator plates are placed prior to the the detector's crystal in such a manner that the photons incident from all but a single direction are blocked by the plates. This guarantees that only photons incident from the desired direction will strike the photon detector.

Brain SPECT has become an important diagnostic and research tool in nuclear medicine. The ultimate value of this procedure depends on good technique in acquisition setup and proper data reconstruction [1 2]. This paper analyzes reconstruction methods combined with pre- and post-filtering for Single Photon Emission Computed Tomography (SPECT) in terms of convergence speed and image quality.

\section{Filtered Backprojection Reconstruction}

An image of the cross section of an object can be recovered or reconstructed from the projection data. In ideal conditions, projections are a set of measurements of the integrated values of some parameter of the object. If the object is represented by a two dimensional function $f(x, y)$ and each line integral by the $(\theta, t)$ parameters, the line integral $P_{\theta}(t)$ is defined as:

$$
P_{\theta}(t)=\int_{-\infty}^{+\infty} \int_{-\infty}^{+\infty} f(x, y) \delta(x \cos \theta+y \sin \theta-t) d x d y
$$

The function $P_{\theta}(t)$ is known as the Radon transform of the function $f(x, y)$. A projection is formed by combining a set of line integrals. The simplest projection is a collection of parallel ray integrals and is given by $P_{\theta}(t)$ for a constant $\theta$. Another type of projection is possible if a single source is placed in a fixed position relative to a line of detectors. This projection is known as fan beam projection because the line integrals are measured along fans.

The key to tomographic imaging is the Fourier Slice Theorem which relates the measured projection data to the two-dimensional Fourier transform of the object cross section. The Fourier Slice Theorem is stated as follows: "The Fourier 
transform $S_{\theta}(w)$ of a parallel projection $P_{\theta}(t)$ of an image $f(x, y)$ taken at angle $\theta$ and defined to be:

$$
S_{\theta}(w)=\int_{-\infty}^{+\infty} P_{\theta}(t) \exp (-j 2 \pi w t) d t
$$

gives a slice of the two-dimensional Fourier transform:

$$
F(u, v)=\int_{-\infty}^{+\infty} \int_{-\infty}^{+\infty} f(x, y) \exp (-j 2 \pi(u x+v y)) d x d y
$$

subtending an angle $\theta$ with the $u$-axis", that is,

$$
S_{\theta}(w)=F(u=w \cos \theta, v=w \sin \theta)
$$

The above result is the essence of straight ray tomography and indicates that by having projections of an object function at angles $\theta_{1}, \theta_{2}, \ldots, \theta_{k}$ and taking the Fourier transform of them, the values of $F(u, u)$ can be determined on radial lines.

In practice only a finite number of projections of an object can be taken. In that case it is clear that the function $F(u, v)$ is only known along a finite number of radial lines so that one must then interpolate from these radial points to the points on a square grid.

The filtered backprojection (FBP) algorithm can be easily derived from the Fourier Slice Theorem. An image of the cross section $f(x, y)$ of an object can be recovered by:

$$
f(x, y)=\int_{0}^{\pi} Q_{\theta}(x \cos \theta+y \sin \theta) d \theta
$$

where

$$
Q_{\theta}(t)=\int_{-\infty}^{+\infty} S_{\theta}(w)|w| \exp (j 2 \pi w t) d w
$$

The FBP algorithm then consists of two steps: the filtering part, which can be visualized as a simple weighting of each projection in the frequency domain, and the backprojection part.

\section{Maximum Likelihood Expectation Maximization (ML-EM)}

In emission tomography, a compound containing a radioactive isotope is introduced into the body and forms an unknown emitter density $\lambda(x, y)$ under the body's functional activity. Emissions then occur according to a Poisson process. The acquisition system usually consists of $D$ detectors so that the measured data $n^{*}(1), \ldots, n^{*}(D)$ represents the counts of photons emitted by the body and measured by each one of the detectors.

The maximum likelihood expectation maximization algorithm (ML-EM) 3456 determine an estimate $\hat{\lambda}$ of $\lambda$ which maximizes the probability 
$p\left(n^{*}(1), \ldots, n^{*}(D) \mid \lambda\right)$ of observing the actual detector count data over all possible densities. Let $n(b)$ represent the number of unobserved emissions in each of $B$ boxes (pixels) partitioning an object containing an emitter and let $p(b, d)$ be the probability of an emission in box $b$ is detected in detector unit $d$. ML-EM is an iterative reconstruction algorithm which starts with an initial estimate $\lambda_{0}$ and gives the new estimate $\hat{\lambda}$ from an old estimate $\hat{\lambda}^{\prime}$ :

$$
\hat{\lambda}(b)=\hat{\lambda}^{\prime}(b) \sum_{d=1}^{D} \frac{n^{*}(d) p(b, d)}{\sum_{b^{\prime}=1}^{B} \lambda^{\prime}\left(b^{\prime}\right) p\left(b^{\prime}, d\right)}
$$

\section{Ordered Subset Expectation Maximization (OSEM)}

The application of Expectation Maximization (EM) algorithms in emission tomography has led to the introduction of many related techniques. While quality of reconstruction is good, the application of EM is computer intensive and its convergence slow. Ordered subset expectation maximization (OS-EM) 7] algorithm for computed tomography groups projection data in ordered subsets. The standard EM algorithm (i.e., projection followed by backprojection) is then applied to each of the subsets in turn. The resulting reconstruction becomes the starting value for use with the next subset. An iteration of the OS-EM algorithm is defined as a single pass through all the specified subsets. Further iterations may be performed by passing through the same ordered subsets, using as a starting point the reconstruction provided by the previous iteration. By selecting mutually exclusive subsets, each OS-EM iteration has a similar computation to a single EM iteration.

In SPECT, the sequential processing of ordered subsets is very natural, as projection data is collected separately for each projection angle (as a camera rotates around the patient in SPECT); counts on single projections can form successive subsets.

\section{$5 \quad$ Prefiltering and Postfiltering}

A major drawback of FBP algorithms for tomographic image reconstruction is the undesired amplification of the high frequency noise and its impact on image quality. These effects are caused by the filtering operation or multiplication of $S_{\theta}(w)$ by $|w|$ in equation 6 . In order to attenuate the high frequency noise amplified during FBP reconstruction, a number of window function has been proposed. In this way, the reconstruction method described by equations 5 and 6 is normally redefined by applying a frequency window which returns to zero as the frequency tends to $\pi$. Among the most common window functions used for FBP reconstruction are: $i$ ) sinc (Shepp-Logan filter), ii) cosine, iii) Hamming and, iv) Hanning window functions. However, even when the reconstruction noise is kept low using a noise controlled FBP approach, the noise captured by the acquisition system needs to be filtered out to improve the quality of reconstructed images. 


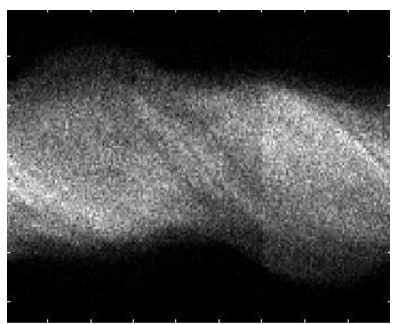

(a)

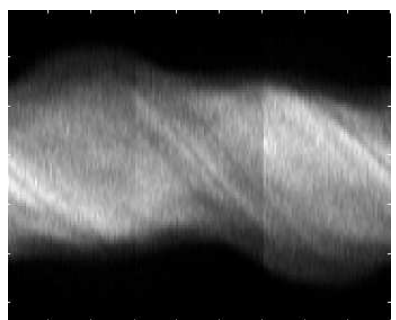

(b)

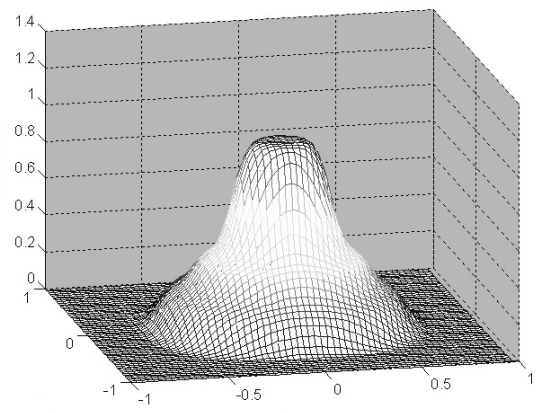

(c)

Fig. 1. a) SPECT sinogram acquired by a three head gammacamera, b) Filtered SPECT data, c) Frequency response of noise filter

Moreover, the preprocessing stage of most automatic SPECT image processing systems often incorporates prefiltering, reconstruction and postfiltering to minimize the noise acquired by the gammacamera as well as the noise amplified during FBP reconstruction.

\section{Image Quality and Performance Evaluation}

Image files were taken from a concurrent study investigating the use of SPECT as a diagnostic tool for the early onset of Alzheimer-type dementia. SPECT data were acquired by a three head gammacamera Picker Prism 3000. The patient is injected with a ${ }^{99 m} \mathrm{Tc}-\mathrm{ECD}$ radiopharmeceutical which emits gamma rays that are detected by the detector. A total of 180 projections were taken with a 2-degree angular resolution.

Fig. 1 shows the effects of noise and filtering on projection data acquired by the gammacamera. The acquired sinogram shows a visible high frequency noise that is effectively filtered by a 2D frequency sampling FIR filter. Fig. 2 shows the effect of noise on FBP image reconstruction and the effectiveness of pre- and post-filtering. Note that, when a simple ramp filter is used, the noise completely 


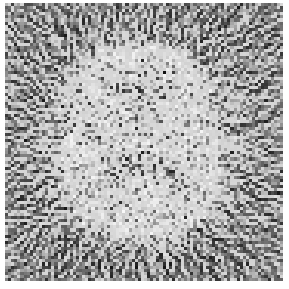

(a)

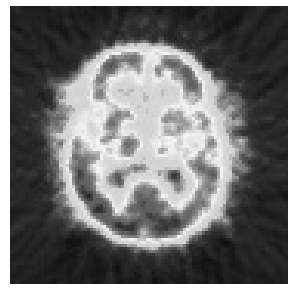

(b)

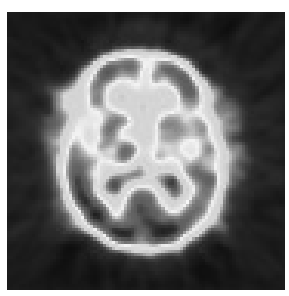

(c)

Fig. 2. FBP reconstruction of a SPECT image with a) RAM-LAK filter, b) with FIR prefiltering, c) with pre- and post-filtering

corrupts the reconstructed image. Pre- and post-filtering improves the quality of FBP reconstruction by removing the huge high frequency noise present in SPECT data and the residual noise after reconstruction. Thus, FBP yields good image quality for analysis and display of SPECT data although residual noise is observed in the images as shown in Fig. 2a.

ML-EM algorithm is an iterative algorithm for image reconstruction that better models the photons emitted by a radioactive source and yields better image quality when compared to FBP. Fig. 3 shows the slow convergence of ML-EM to a final reconstructed image. It is shown that the iterative algorithm described by equation 7 requires from 15 to 20 iterations to converge and yields a final image with improved quality when compared to FBP as shown in Fig. 4.

OS-EM image reconstruction, that groups projection data in ordered subsets and performs projection followed by backprojection to each of the subsets in turn, yields an acceleration on the convergence of the image reconstruction process. Thus, with a number of subset equal to the number of iterations required by

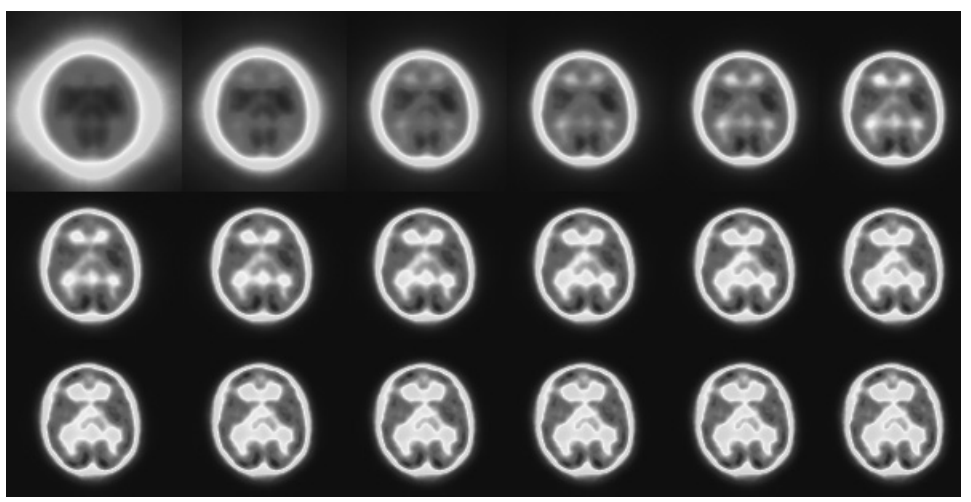

Fig. 3. Convergence of ML-EM SPECT image reconstruction 


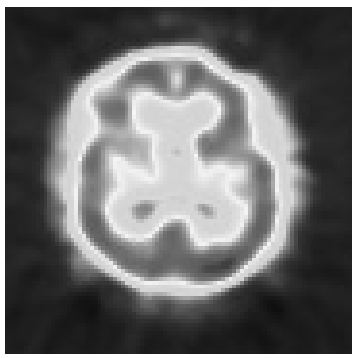

(a)

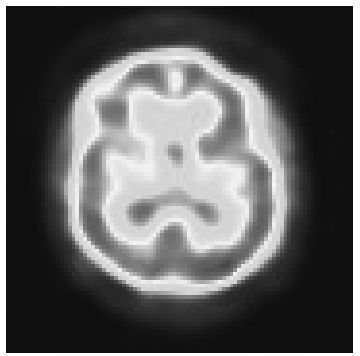

(b)

Fig. 4. Comparison of FBP and ML-EM methods for SPECT image reconstruction. a) FBP, b) ML-EM.

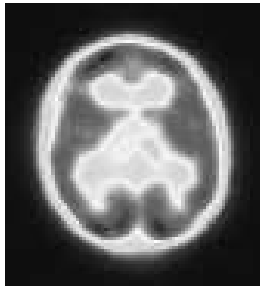

(a)

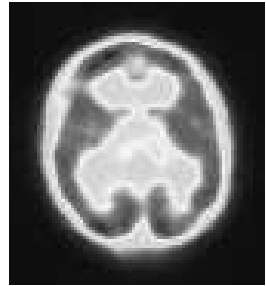

(b)

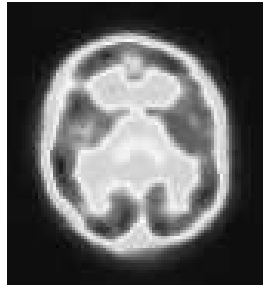

(c)

Fig. 5. Result after one OSEM iteration. Image reconstruction partitioning the set of detectors into a) 10, b) 15 and c) 20 subsets.

ML-EM to converge, OS-EM converges in a single iteration performed over all the subsets. Thus, for the example shown in Fig. 3, OS-EM should converge by partitioning the whole set of detector elements into about 15-20 subsets. Fig. 5 shows the results of a single iteration performed by the OS-EM algorithm with a different number of subsets. It is clearly shown that with 15 and 20 subsets the image appear to be of similar quality to ML-EM with the advantage of a speedup of the order of the number of subsets.

\section{Conclusions}

Classical filtered backprojection and statistical maximum likelihood expectation maximization image reconstruction algorithms were evaluated in terms of image quality and processing delay. Image files were taken from a concurrent study investigating the use of SPECT as a diagnostic tool for the early onset of Alzheimer-type dementia. FBP image reconstruction needs a careful control and the noise since it tends to amplify high frequency noise. Pre- and post-filtering improves the quality of FBP reconstruction by removing the huge high frequency 
noise present in SPECT data and the residual noise after reconstruction. MLEM yields better image quality when compared to FBP since a precise statistical model of the emission is used. However, the processing delay is considerable due to its slow convergence. OS-EM was found to be a good trade-off between image quality and processing delay since it converges in a single iteration by partitioning the set off detection elements into about 15-20 subsets.

Acknowledgments. This work has been funded by the PETRI project DENCLASES (PET2006-0253) of the Spanish MEC and the regional Excellence Project (TIC-02566) of the Consejería de Innovación Ciencia y Empresa (Junta de Andalucía, Spain). We also acknowledge financial support by the German Academic Exchange Service (DAAD).

\section{References}

1. Vandenberghea, S., D'Asselera, Y., de Wallea, R.V., Kauppinenb, T., Koolea, M., Bouwensa, L., Laerec, K.V., Lemahieua, I., Dierckx, R.: Iterative reconstruction algorithms in nuclear medicine. Computerized Medical Imaging and Graphics, 105111 (2001)

2. Bruyant, P.P.: Analytic and iterative reconstruction algorithms in spect. The Journal of Nuclear Medicine 43, 1343-1358 (2002)

3. Shepp, L.A., Vardi, Y.: Maximum likelihood reconstruction for emission tomography. IEEE Transactions on Medical Imaging MI-1, 113-122 (1982)

4. Vardi, Y., Shepp, L.A., Kaufman, L.: A statistical model for positron emission tomography. Journal of the American Statistical Association 80, 8-20 (1985)

5. Lange, K., Carson, R.: Em reconstruction for emission and transmission tomography. Journal of Computer Assisted Tomography 8, 306-312 (1984)

6. Chornoboy, E.S., Chen, C.J., Miller, M.I., Miller, T.R., Snyder, D.L.: An evaluation of maximum likelihood reconstruction for spect. IEEE Transactions on Medical Imaging 9, 99-110 (1990)

7. Hudson, H.M., Larkin, R.S.: Accelerated image reconstruction using ordered subsets of projection data. IEEE Transactions on Medical Imaging 13, 601-609 (1994) 